\title{
Validation of alternate modes of administration of the lung function questionnaire (LFQ) in subjects with smoking history
}

This article was published in the following Dove Press journal: International Journal of Chronic Obstructive Pulmonary Disease 7 December 2010

Number of times this article has been viewed

\author{
Anand A Dalal' \\ Carla DeMuro-Mercon² \\ Sandy Lewis ${ }^{2}$ \\ Lauren Nelson ${ }^{2}$ \\ Theresa Gilligan ${ }^{2}$ \\ Lori McLeod ${ }^{2}$ \\ 'United States Health Outcomes, \\ GlaxoSmithKline, Durham, NC, USA; \\ ${ }^{2}$ Patient-Reported Outcomes, RTI \\ Health Solutions, Research Triangle \\ Park, NC, USA
}

Purpose: The Lung Function Questionnaire (LFQ) was developed and validated as a case-finding tool to identify patients at risk of airflow obstruction (AO) that should be evaluated further using spirometry. Our objective was to assess the usability and validity of additional questionnaire-administration modes, including Web-based, interactive voice response system (IVRS)-based, and interviewer-based modes.

Design: This multicenter, prospective, noninterventional data-collection study enrolled 149 individuals aged $\geq 40$ years with current or former smoking history. A two-visit crossover design was employed; patients completed the paper-based LFQ and were randomly assigned to complete one of three alternate modes.

Methods: Statistical evaluation included item-level, scale-level, and AO risk-classification comparisons; a satisfaction survey assessed patient preference.

Results: This study showed a great degree of concordance between alternate forms of the LFQ and the paper version. Results indicated an absence of floor and ceiling effects and the average LFQ item-level means were consistent across modes. LFQ scores were stable between assessments, (administered approximately one week apart) showed exceptionally good agreement, and AO risk classification using the LFQ cut point was consistent across modes.

Conclusions: The LFQ is an important case-finding tool to aid primary care physicians in further evaluating symptomatic patients at risk of AO. The alternate modes will further facilitate the implementation and widespread uptake of this tool.

Keywords: COPD, spirometry, screening, PRO, case-finding, LFQ

\section{Introduction}

Chronic obstructive pulmonary disease (COPD) is an extremely debilitating disease characterized by limitation in lung airflow due to lung damage that is not completely reversible. ${ }^{1}$ The disorder is currently the fourth leading cause of chronic morbidity and mortality in the United States, and a recent study suggests that it will rank fifth in disease burden worldwide by $2020 .^{2}$ Although nonsmokers can develop COPD, tobacco smoking is the primary risk factor for developing the disease. Currently, clinical diagnosis of the disorder is based on the amount of airway limitation present as measured by spirometry. Typically, pulmonary function tests are not conducted, which contributes to the wide underdiagnosis of COPD. ${ }^{3,4}$ This delayed diagnosis may lead to delayed treatment and poorer patient outcomes.

To combat the problem of underdiagnosis, the Lung Function Questionnaire (LFQ) was developed as a case-finding tool to identify patients at risk of airflow obstruction (AO) that should be evaluated further using spirometry. The tool contains five items
Correspondence: Anand Dalal GlaxoSmithKline, 5 Moore Drive, Mail Stop 17.1355B, Durham, NC 27709, USA

Tel + I 9194837286

$\mathrm{Fax}+19194830103$

Email anand.a.dalal@gsk.com 
that measure COPD symptoms and risk factors such as age and smoking status. The LFQ was developed and originally validated using a paper-based $(\mathrm{P})$ mode of administration in a cross-sectional study at two primary care offices in which screening properties, scoring, and other psychometrics were explored. ${ }^{2}$ In a subsequent study, the LFQ (paper mode) was also administered in the waiting rooms of 36 primary care practices in the United States to evaluate the prevalence of AO as measured by the LFQ and confirmed by spirometry. ${ }^{5}$ The LFQ was shown to be a helpful AO case-finding tool with an estimated COPD prevalence of $17.9 \%$ in the target sample of smokers. Given the previous studies' success in earlier case identification using the LFQ, the objective of the current study was to further the development, acceptance, and accessibility of the LFQ by validating additional modes of questionnaire administration, including Web-based (W-based), interactive voice response system (IVRS)-based, and interviewer-based (I-based) modes.

\section{Methods}

\section{Study design}

This multicenter, prospective, non-interventional data collection was conducted in September 2009 with 149 individuals aged 40 years and older with current or former smoking history (self report of smoking status). A two-visit, crossover design was used. In addition to completing the paper-based mode of the LFQ (see Figure 1), participants were randomly assigned to complete one of the three alternate modes of the LFQ along with a satisfaction survey designed to ascertain participants' acceptance of and preference for the different LFQ administration modes. Participants were assigned to one of six sequence groups based on the LFQ mode completed and order of administration (ie, paper-Web [P-W], paperIVRS [P-IVRS], paper-interviewer [P-I], Web-paper [W-P], IVRS-paper [IVRS-P], and interviewer-paper [I-P]) at the two visits (Figure 2). The IVRS was commissioned through Cisys LifeSciences and the Web version was developed in OutcomeLogix by Maaguzi LLC. The P, and W versions all used the visual format provided in Figure 1. To ensure continuity, teams of trained interviewers at both qualitative research settings facilitated administration of each mode of the LFQ at visit 1 and visit 2. Scripts for the IVRS and I versions are available upon request to the corresponding author.

\section{Subject recruitment and enrollment criteria}

Qualitative research firms in Pennsylvania and North Carolina were selected to identify and recruit appropriate study participants. Study participants were required to meet the following criteria:

- 40 years of age or older

- Current or former smoker (defined as $\geq 10$ pack-years) (self-report)

- Able to provide informed consent and can read and understand English

- No diagnosis of COPD, emphysema, or asthma (obtained via self-report)

The qualitative research sites identified subjects with a self-reported smoking history from their records and advertised the study in an effort to attain diversity among participants. Recruitment through qualitative sites allowed for greater access to a broader range of potential participants from diverse ethnic, geographic, educational, and socioeconomic backgrounds. It also assisted in avoiding any bias related to health care-seeking individuals, especially given the nature of the underdiagnosis of COPD and the denial of health problems that often accompany individuals who smoke. The qualitative sites conducted a prescreen by telephone, and eligible subjects who gave written consent to participate completed the LFQ and provided demographic and medical history information. Each site recruited approximately 75 subjects for a target enrollment of 150 participants. The total number of participants actually enrolled was 149 . Two participants did not complete either the paper or an alternate LFQ mode, yielding 147 participants in this study. Each participant was asked to complete two study visits. The study was approved by a central institutional review board.

\section{Study assessments Questionnaires}

At each visit, participants were instructed to complete a version of the LFQ (either paper or an alternate mode). Each item on the LFQ has five possible responses, coded from 1 to 5 . The five items are summed to provide a total LFQ score, which can range from 5 to 25, with lower scores indicating greater risk of $\mathrm{AO}$ (see Figure 1). In addition to administering the LFQ, demographic information, general health status, presence of active colds or infections, focused respiratory-related medical history, activity limitations, smoking history, and information about computer knowledge and experience were collected at visit 1 (see Figure 2). At visit 2 (approximately 7 days after visit 1), participants were asked to complete the LFQ, questions about their general health status and presence of active colds or infections, and satisfaction surveys about the different modes of the LFQ. The satisfaction surveys were created to assess participants' overall experience with 


\section{Lung Function Questionnaire \\ Do you suffer from breathing problems and/or frequent cough?}

These questions ask about your breathing problems and/or frequent cough. As you answer these questions, please think about how you are feeling physically when you are experiencing these symptoms. For each question, choose the one answer that best describes your symptoms. Share the answers with your doctor.

Step 1: Answer each question by making a mark $(x)$ and write the score in the box provided next to it.

Step 2: Add the score boxes for your total score

Step 3: Take the test to the doctor to talk about your score

1. How often do you cough up mucus?
Never
(5) Rarely
(4)
Sometimes (3)
Often
(2) Very Often 1

2. How often does your chest sound noisy (wheezy, whistling, rattling) when you breathe?

\begin{tabular}{ll|l|l|l|l|l|l|l|} 
Never (5) Rarely (4) Sometimes (3) Often & (2) & Very Often (1) \\
\hline
\end{tabular}

3. How often do you experience shortness of breath during physical activity

(walking up a flight of stairs

or walking up an incline without stopping to rest)?

\begin{tabular}{ll|l|l|l|l|l|l|} 
Never (5) Rarely (4) Sometimes (3) & Often & (2) & Very Often (1)
\end{tabular}

4. How many years have you smoked?

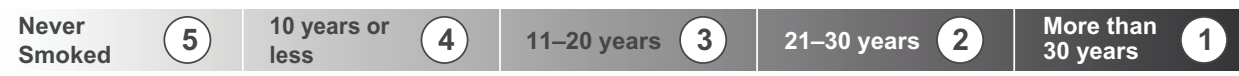

5. What is your age?

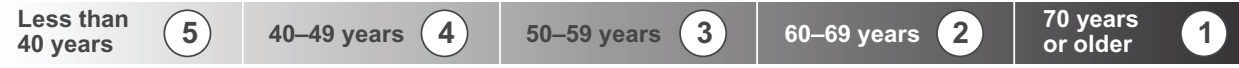

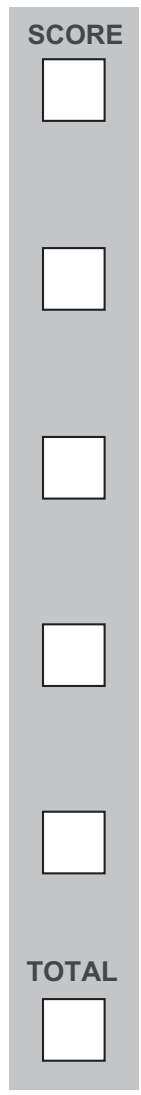

Step 4: If your score is 18 or less then you may be at greater risk for Chronic Obstructive Pulmonary Disease (COPD) which includes chronic bronchitis, emphysema or both. Ask your doctor if you need a simple breathing test. This questionnaire is intended to determine your risk of COPD. No matter what your score, you should still talk to your doctor about your symptoms.

Figure I LFQ (paper and Web format).

and preference for the alternate modes of administration of the LFQ. Participants were asked to provide feedback on the paper-based mode of the LFQ and the alternate mode of administration they completed. Questions assessed the instructions for completing the questionnaire, any difficulty experienced when completing the questionnaire, and a rating of their overall experience with completing the questionnaire. Response categories for these questions ranged from 


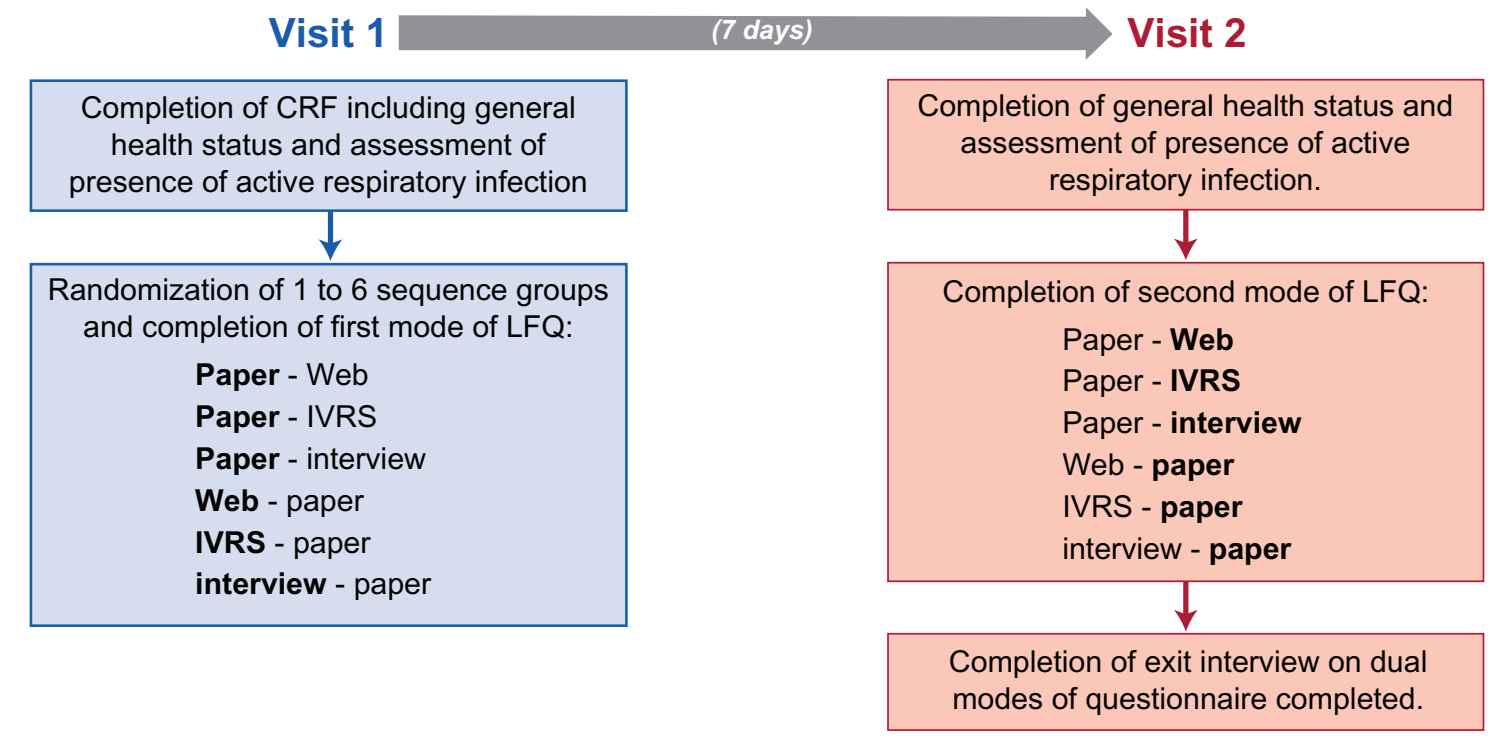

Figure 2 Study flow diagram.

Abbreviations: IVRS, interactive voice response system; LFQ, Lung Function Questionnaire.

0 ("terrible") to 10 ("excellent"). Participants were also asked to indicate their preferred mode of administration and provide a brief rationale for their choice.

\section{Statistical analysis and evaluation}

Only those participants who completed all questions of two LFQ modes (one of which was always paper) were included in the analysis. Additionally, participants who reported a change in status regarding either an active cold or infection at visit 1 and visit 2 were omitted from the analysis because of the potential influence of health changes on their LFQ responses. The statistical evaluation was conducted separately by the six sequence groups and across the three pairs of alternative LFQ modes (ie, P-W and W-P sequences were combined to form a single paper- and Web-based grouping (P/W); P-IVRS and IVRS-P form P/IVRS; and P-I and I-P form $\mathrm{P} / \mathrm{I}$ ). These analyses included item-level, scale-level, and $\mathrm{AO}$ risk classification comparisons. In addition, the satisfaction survey and exit interview provided input related to patient preference for the different modes.

\section{Item-level}

For the item-level assessments, responses for each LFQ item were compared across the three pairs of alternate LFQ modes (ie, P/W, P/IVRS, P/I) and between each of the six sequence groups (ie, P-W, P-IVRS, P-I, W-P, IVRS-P, I-P) to identify any potential response anomalies, such as floor and ceiling effects or a restricted range of responses. In addition, agreement of responses across mode was assessed. To measure agreement, item-level weighted kappas were computed for the three pairs of LFQ modes and separately by the six sequence groups. Weighted kappa is a recommended approach for ordered categorical responses because it provides partial credit for agreement for responses that differ by only one or two categories versus only crediting equivalent responses as agreement. ${ }^{6}$ Landis and $\mathrm{Koch}^{7}$ suggest guidelines for assessing the magnitude of kappa. A kappa ranging from 0.21 to 0.40 is considered poor to fair, 0.41 to 0.60 is moderate, 0.61 to 0.80 is substantial, and over 0.81 is nearly perfect.

\section{Scale-level}

At the scale level, overall LFQ scores were computed and compared for the six sequence groups and three pairs of modes. In addition, test-retest reliability analyses were run between the paper-based mode and the three alternate LFQ administration modes.

Before comparing the LFQ modes, we tested whether a statistically significant sequence effect was observed. This effect is important to consider because a participant's responses should not depend on which mode they received first. To evaluate the sequence effect, we computed the differences for each participant between the first and second administrations and using a between-groups $t$-test, compared those who received the paper-based mode first to those who received the paper-based mode at the final administration. A $P$ value $\leq 0.05$ for this test was supportive of a statistically significant sequence effect.

Following nonsignficant sequence effects, further analyses were conducted on the three pairs of LFQ modes (ie, 
$\mathrm{P} / \mathrm{W}, \mathrm{P} / \mathrm{IVRS}, \mathrm{P} / \mathrm{I})$ to test for differences in response by mode. The difference in the mean scores for each of the three pairs of LFQ modes was computed and a paired $t$-test was performed (ie, where the null hypothesis is zero). A $P$ value $\leq 0.05$ the difference in the means was statistically significant from zero.

Additionally, concordance between the paper-based mode and the three alternate LFQ administration modes was measured by computing the intraclass correlation coefficient (ICC) using a one-way random effects analysis of variance (ANOVA). Separate ICC estimates were computed for each pair of modes (ie, P/W, P/IVRS, P/I) and compared to previous estimates of the test-retest reliability for the paper-based LFQ. It is recommended that ICCs be at least 0.70 for multi-item scales. ${ }^{8}$

\section{Airway obstruction risk classification}

In the previous validation study, the LFQ Working Group selected a score of 18 to indicate a great risk of $\mathrm{AO}$, leaning toward greater sensitivity in an effort to minimize missing patients with $\mathrm{AO} .^{2}$ For the present study, this same cut point was applied to the scores on both the paper-based and the alternate mode, and the percent agreement in classification (ie, likely obstructed versus not likely obstructed) and kappa agreement were computed.

\section{Patient satisfaction}

Patient acceptance and satisfaction with answering the LFQ questions via the different modes was assessed. Responses to the satisfaction survey were compared based on mode of administration. Participants were encouraged to share spontaneous feedback during the in-person visits, and an open-ended question was included at the end of the satisfaction survey to illicit a rationale for preference choice of one mode over another or other issues that should be addressed before future use of each mode.

\section{Results}

\section{Study sample}

Most participants in this study were white $(81.0 \%)$, between the ages of 40 to 50 years (46.3\%), and had at least some college experience (Bachelor's degree, 29.3\%). Table 1 shows the

Table I Sample demographics at first administration, overall and by mode of administration

\begin{tabular}{|c|c|c|c|c|}
\hline \multirow[t]{2}{*}{ Characteristic } & \multirow{2}{*}{$\begin{array}{l}\text { Overall } \\
(n=147)\end{array}$} & \multicolumn{3}{|c|}{ Alternate mode of administration } \\
\hline & & $\begin{array}{l}\text { Web } \\
(n=5 I)\end{array}$ & $\begin{array}{l}\text { IVRS } \\
(n=47)\end{array}$ & $\begin{array}{l}\text { Interviewer } \\
(n=49)\end{array}$ \\
\hline \multicolumn{5}{|l|}{ Gender, n (\%) } \\
\hline Male & 72 (49.0\%) & $26(51.0 \%)$ & $26(55.3 \%)$ & $20(40.8 \%)$ \\
\hline Female & $75(51.0 \%)$ & $25(49.0 \%)$ & $21(44.7 \%)$ & $29(59.2 \%)$ \\
\hline Age: mean (SD) & $53.4(8.9)$ & $53.1(9.9)$ & $53.2(8.1)$ & $53.9(8.8)$ \\
\hline \multicolumn{5}{|l|}{ Age, n (\%) } \\
\hline 40-50 years & $68(46.3 \%)$ & $25(49.0 \%)$ & $22(46.8 \%)$ & $21(42.9 \%)$ \\
\hline $51-60$ years & $42(28.6 \%)$ & $12(23.5 \%)$ & $16(34.0 \%)$ & $14(28.6 \%)$ \\
\hline$>60$ years & $37(25.2 \%)$ & 14 (27.5\%) & $9(19.2 \%)$ & $14(28.6 \%)$ \\
\hline \multicolumn{5}{|l|}{ Race/Ethnicity, n (\%) } \\
\hline Black or African American & $21(14.3 \%)$ & 7 (I4.0\%) & $6(12.8 \%)$ & $8(16.3 \%)$ \\
\hline White or Caucasian & $119(81.0 \%)$ & 41 (80.0\%) & $39(83.0 \%)$ & $39(79.6 \%)$ \\
\hline White and American & $4(2.7 \%)$ & I (2.0\%) & $2(4.3 \%)$ & I (2.0\%) \\
\hline \multicolumn{5}{|l|}{ Indian/Alaska Native } \\
\hline Hispanic or Latino & $2(1.4 \%)$ & $2(4.0 \%)$ & $0(0.0 \%)$ & $0(0.0 \%)$ \\
\hline Asian, Native Hawaiian, & I (0.7\%) & $0(0.0 \%)$ & $0(0.0 \%)$ & I $(2.0 \%)$ \\
\hline \multicolumn{5}{|l|}{ Pacific Islander, or Other } \\
\hline \multicolumn{5}{|l|}{ Education, n (\%) } \\
\hline Less than HS diploma & I (0.7\%) & $0(0.0 \%)$ & $\mathrm{I}(2.1 \%)$ & $0(0.0 \%)$ \\
\hline HS diploma/GED & 37 (25.2\%) & 15 (29.4\%) & $9(19.2 \%)$ & $13(26.5 \%)$ \\
\hline Some college & $30(20.4 \%)$ & $10(19.6 \%)$ & I I (23.4\%) & $9(18.4 \%)$ \\
\hline Associate's degree & 19 (12.9\%) & 7 (13.7\%) & $6(12.8 \%)$ & $6(12.2 \%)$ \\
\hline Bachelor's degree & 43 (29.3\%) & 16 (31.4\%) & 12 (25.5\%) & 15 (30.6\%) \\
\hline Master's degree & $15(10.2 \%)$ & $3(5.9 \%)$ & $6(12.8 \%)$ & $6(12.2 \%)$ \\
\hline Professional degree & $0(0.0 \%)$ & $0(0.0 \%)$ & $0(0.0 \%)$ & $0(0.0 \%)$ \\
\hline Doctoral degree & $2(1.4 \%)$ & $0(0.0 \%)$ & $2(4.3 \%)$ & $0(0.0 \%)$ \\
\hline Prior spirometry, n (\%) & 48 (32.7\%) & 17 (33.3\%) & 17 (36.2\%) & I 4 (28.6\%) \\
\hline BMI, mean (SD) & $28.7(5.8)$ & $28.3(5.6)$ & $28.5(6.4)$ & $29.4(5.5)$ \\
\hline
\end{tabular}

Abbreviations: BMI, body mass index; GED, general education degree; HS, high school; IVRS, interactive voice response system; SD, standard deviation. 
characteristics of the overall study sample, and separately by the three alternate modes of administration (ie, W-, IVRS-, or I-based). Approximately 33\% of the participants reported having prior spirometry tests. The average body mass index (BMI) in this sample was in the range that is generally considered to be overweight $(\mathrm{BMI}=25.00$ to 29.99$) .{ }^{9}$ The characteristics of participants assigned to the W-, IVRS-, or I-based administration modes were comparable across all characteristics.

As anticipated based on the enrollment criteria, over $60 \%$ of the participants reported being current smokers. Among those assigned to the W-based mode, slightly more reported being a former smoker than those taking the IVRS- and I-based modes. Across both current and former smokers, the average number of cigarettes reported smoked per day was 19.7 and the average duration of smoking was approximately 23 years. The number of pack-years was computed using the average number of cigarettes and the average number of years smoked. General health reports were very similar overall and by mode of administration (Table 2). Acute respiratory illness was not commonly reported, with less than $10 \%$ of the participants reporting a cold at the time of either study visit.

With respect to computer experience, most participants reported having a home computer and Internet access. Additionally, over $80 \%$ of the participants reported either understanding most of their computer's software and having little trouble learning new software or completely understanding software.
Twelve participants reported active colds or infections at only one assessment indicating a change in their respiratory state and were not included in the main psychometric analysis, yielding 135 participants.

Item-level

The evaluation of the item response distributions provided no evidence of ceiling or floor effects. The modal response for each pair of LFQ administrative modes was typically either identical or adjacent response categories. For example, for the item 1 (cough up mucus) P- and W-based modes, both have modal responses in category 4 , with $54.2 \%$ of participants responding. This finding indicates that, in general, participants responded similarly at the two administrations.

Item level descriptive statistics are comparable across each pair of administration modes with the P-and W-based pair showing the highest level of consistency. Few participants reported symptom items 1 through 3 , the mean score ranged from 3.1 to 4.1 . The mean number of years smoked (item 4) ranged from 2.2 to 2.4, corresponding to an average greater than 25 years; while the mean score for item 5 (age) ranged from 3.0 to 3.2, indicating that the average age was in the range 50 to 59 years. A similar pattern was also present across the six sequence groups.

Figure 3 displays a bar chart plot of the LFQ item level means for each mode of the LFQ. The modes have similar magnitude of item level means, with slightly lower means for participants assigned to the I-based mode, indicating that

Table 2 General health questions at both administrations, overall and by mode of administration

\begin{tabular}{|c|c|c|c|c|}
\hline \multirow[t]{2}{*}{ General health } & \multirow{2}{*}{$\begin{array}{l}\text { Overall } \\
(n=147)\end{array}$} & \multicolumn{3}{|c|}{ Alternate mode of administration } \\
\hline & & $\begin{array}{l}\text { Web } \\
(n=5 I)\end{array}$ & $\begin{array}{l}\text { IVRS } \\
(n=47)\end{array}$ & $\begin{array}{l}\text { Interviewer } \\
(n=49)\end{array}$ \\
\hline \multicolumn{5}{|c|}{$\begin{array}{l}\text { Would you say that in general } \\
\text { your health is? } \mathrm{n}(\%)\end{array}$} \\
\hline \multicolumn{5}{|c|}{$\mathrm{I}=$ Excellent } \\
\hline First administration & $18(12.2)$ & $5(9.8 \%)$ & $6(12.8 \%)$ & $7(14.3 \%)$ \\
\hline Final administration & $15(10.2 \%)$ & $4(7.8 \%)$ & $6(12.8 \%)$ & $5(10.2 \%)$ \\
\hline \multicolumn{5}{|l|}{$2=$ Very good } \\
\hline First administration & $69(46.9 \%)$ & $26(51.0 \%)$ & $23(48.94 \%)$ & $20(40.8 \%)$ \\
\hline Final administration & $72(49.0 \%)$ & $31(60.8 \%)$ & $23(48.94 \%)$ & $18(36.7 \%)$ \\
\hline \multicolumn{5}{|l|}{$3=$ Good } \\
\hline First administration & $54(36.7 \%)$ & $18(35.3 \%)$ & $16(34.0 \%)$ & $20(40.8 \%)$ \\
\hline Final administration & $5 \mathrm{I}(34.7 \%)$ & $14(27.5 \%)$ & $16(34.0 \%)$ & 21 (42.9\%) \\
\hline \multicolumn{5}{|l|}{$4=$ Fair } \\
\hline First administration & $5(3.4 \%)$ & $2(3.9 \%)$ & $2(4.3 \%)$ & I (2.0\%) \\
\hline Final administration & $8(5.4 \%)$ & $2(3.9 \%)$ & $2(4.3 \%)$ & $4(8.2 \%)$ \\
\hline \multicolumn{5}{|l|}{$5=$ Poor } \\
\hline First administration & I (0.7\%) & $0(0.0 \%)$ & $0(0.0 \%)$ & I (2.0\%) \\
\hline Final administration & I (0.7\%) & $0(0.0 \%)$ & $0(0.0 \%)$ & I (2.0\%) \\
\hline
\end{tabular}

Abbreviation: IVRS, interactive voice response system. 


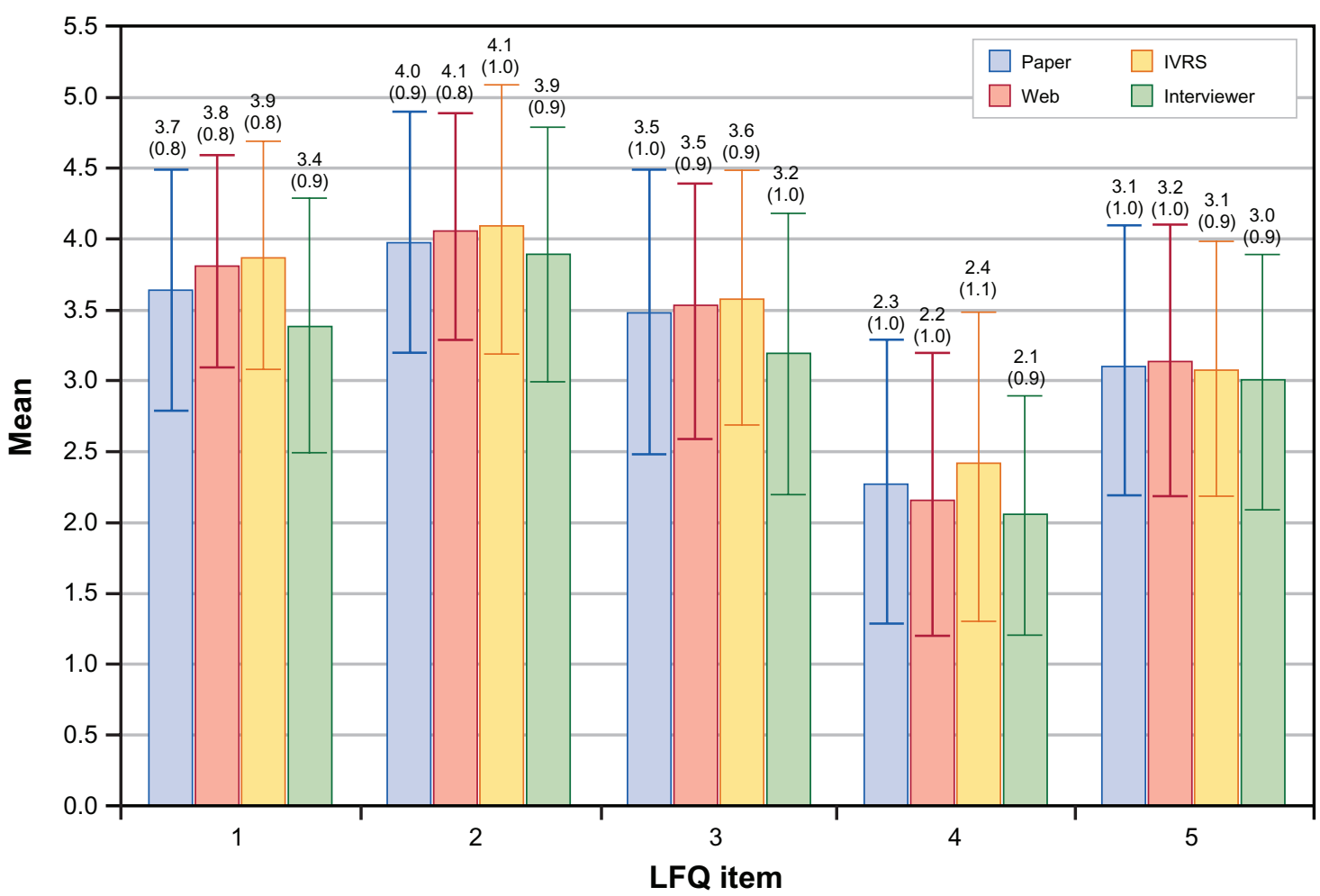

Figure 3 Mean of LFQ items by mode of administration.

Abbreviations: IVRS, interactive voice response system; LFQ, Lung Function Questionnaire.

those participants reported slightly more frequent symptoms of obstruction.

Table 3 contains the kappa statistic and corresponding 95\% confidence interval (CI) as a measure of agreement between LFQ item-level responses of the P-based and alternate modes. The kappa statistics are highly satisfactory, with the lowest observed for shortness of breath between the P- and IVRS-based modes, and the highest estimated for age for the P- and W-based modes and the $\mathrm{P}$ - and I-based modes. Comparable results also were shown by sequence.

\section{Scale-level}

Table 4 shows the descriptive statistics for the LFQ total scores for the three pairs of LFQ modes (ie, P/W, P/IVRS, P/I). The scores ranged from a minimum of 8.0 to a maximum of 23.0 .
The means and medians for all administration modes were less than 18 points, the cut point used in previous studies for indicating a greater risk of obstruction. Notably, the SDs were comparable across the various modes, ranging from a low of 2.2 to a high of 2.8. Participants given the $\mathrm{P} / \mathrm{I}-$ based modes had the lowest LFQ means and the largest score spread; participants assigned to the $\mathrm{P} / \mathrm{W}$-based pair had comparable means; and participants given the P/IVRS-based modes had the highest means.

No significant differences between the paper and any of the alternate modes order of administration were found (ie, P-W, P-IVRS, P-I, W-P, IVRS-P, and I-P). Hence, there was no evidence of a sequence effect, and all further analyses were combined over sequence, yielding three separate analyses, one for each of the three pairs of LFQ modes (ie, P/W, P/IVRS, P/I).

Table 3 LFQ item-level kappa statistics by mode pair

\begin{tabular}{llll}
\hline LFQ Item & $\begin{array}{l}\text { P- and W-based } \\
\text { Kappa }(\mathbf{9 5 \%} \mathbf{~ C l})\end{array}$ & $\begin{array}{l}\text { P-and IVRS-based } \\
\text { Kappa (95\% Cl) }\end{array}$ & $\begin{array}{l}\text { P-and I-based } \\
\text { Kappa (95\% Cl) }\end{array}$ \\
\hline I (cough up mucus) & $0.85(0.75,0.95)$ & $0.65(0.45,0.85)$ & $0.79(0.62,0.96)$ \\
2 (chest noisy) & $0.80(0.69,0.92)$ & $0.78(0.64,0.92)$ & $0.83(0.70,0.95)$ \\
3 (shortness of breath) & $0.76(0.65,0.87)$ & $0.63(0.42,0.83)$ & $0.73(0.57,0.89)$ \\
4 (years smoked) & $0.94(0.88,0.99)$ & $0.89(0.82,0.96)$ & $0.85(0.71,0.98)$ \\
5 (age) & $0.99(0.97,1.00)$ & $0.97(0.94,1.00)$ & $0.99(0.96,1.00)$ \\
\hline
\end{tabular}

Abbreviations: $\mathrm{Cl}$, confidence interval; I, interviewer; IVRS, interactive voice response system; LFQ, Lung Function Questionnaire; P, paper; W, Web. 
Table 4 LFQ total score descriptive statistics by administration mode pair

\begin{tabular}{lllllll}
\hline Mode & N & Mean & SD & Median & Minimum & Maximum \\
\hline P & 48 & 17.0 & 2.4 & 17.0 & 10.0 & 22.0 \\
W & 48 & 16.8 & 2.2 & 17.0 & 11.0 & 21.0 \\
P & 45 & 17.0 & 2.5 & 17.0 & 12.0 & 23.0 \\
IVRS & 45 & 17.2 & 2.6 & 17.0 & 12.0 & 22.0 \\
P & 42 & 15.7 & 2.8 & 16.0 & 8.0 & 20.0 \\
I & 42 & 15.6 & 2.6 & 15.0 & 8.0 & 20.0
\end{tabular}

Abbreviations: I, interviewer; IVRS, interactive voice response system; P, paper; $\mathrm{W}, \mathrm{Web} ; \mathrm{SD}$, standard deviation.

Figure 4 demonstrates the relationship between the LFQ total scores and mode in six boxplots - one for each of the three pairs of LFQ modes. In general, the means and spread of each pair of LFQ modes are highly comparable. Somewhat more variability and negative skewness can be observed in the scores of the P-based and I-based administration than in the other administration mode pairs. However, the maximum score for both the $\mathrm{P}$ - and I-based modes was approximately 20 , indicating that these participants are more likely to be at risk for $\mathrm{AO}$ based on the LFQ.

The mean difference between the P- and W-based scores was $0.2(\mathrm{SD}=0.8)$, yielding a nonsignificant $t$-statistic $(P=0.2)$. For the $\mathrm{P}$ - and IVRS-based scores, the mean difference was $-0.1(\mathrm{SD}=1.6)$ and the $t$-statistic was nonsignificant $(P=0.6)$. The $\mathrm{P}$ - and I-based mean difference was also close to small at $0.1(\mathrm{SD}=1.3)$ and nonsignificant $(P=0.8)$. These results provide evidence that the difference in the LFQ mode means was not statistically different from zero.

As shown in Table 5, the ICCs across mode were higher than the recommended threshold of 0.70 . Notably, the minimum point on the $95 \%$ CI is very close to 0.70 , while the

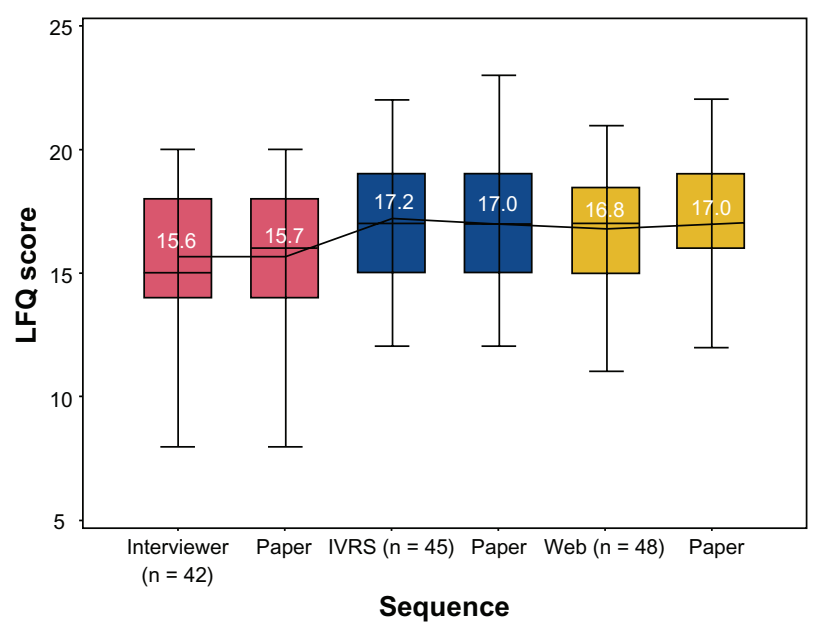

Figure 4 Boxplots of LFQ total score by mode pair.

Abbreviations: IVRS, interactive voice response system; LFQ, Lung Function Questionnaire.
Table 5 ICCs between paper and the alternate mode of administration by mode pair

\begin{tabular}{ll}
\hline $\begin{array}{l}\text { P-based with alternate } \\
\text { mode of administration }\end{array}$ & ICC (95\% CI) \\
\hline W & $0.93(0.88-0.96)$ \\
IVRS & $0.8 I(0.68-0.89)$ \\
I & $0.88(0.79-0.93)$ \\
\hline
\end{tabular}

Abbreviations: $\mathrm{Cl}$, confidence interval; I, interviewer; IVRS, interactive voice response system; ICC, intraclass correlation coefficient; P, paper; W, Web.

maximum is near or above 0.90 . This provides evidence that the LFQ total scores were stable over the 7 days between assessments. In addition, the P-based and alternate administration modes show strong agreement.

\section{Airway obstruction risk classification}

Table 6 shows the percent agreement in classification (ie, likely obstructed versus not likely obstructed). Overall, the classifications were highly comparable across modes of administration. For the P-and W-based comparisons, approximately $71 \%$ of the sample was considered at risk of AO and $25 \%$ were considered not at risk by both modes. Only 2 participants were classified as having airway obstruction risk under the W-based mode but were classified as not at risk for obstruction with the P-based mode. The kappa statistic was highly satisfactory at 0.89 . The P- and IVRS-based administration modes classified $62.2 \%$ participants as having a higher risk of $\mathrm{AO}$ and 12 participants as not at risk, yielding a high overall agreement of approximately $89 \%$. Notably, only $11.1 \%$ of participants were classified differently between these modes. For these the P-based mode classified them as being at risk and the IVRS-based mode did not. Nevertheless, the kappa statistic indicates good agreement overall. The P-based mode and the I-based mode resulted in the lowest kappa, but had a very high percentage of overall agreement at $85.7 \%$. Most participants $(73.8 \%)$ were classified as at risk or not at risk (11.9\%) by both the P- and I-based modes. However, 6 participants were misclassified.

Given the Landis and Koch guidelines, the agreement in classification is nearly perfect for $\mathrm{P} / \mathrm{W}$, substantial for P/IVRS and moderate for $\mathrm{P} / \mathrm{I}$.

\section{Patient satisfaction}

Although the LFQ administrations were not timed, the trained interviewers reported that participants completed the LFQ in less than 5 minutes; with the IVRS requiring slightly more time and the $\mathrm{W}$ - and P-based versions requiring slightly less time to complete, on average. Approximately $60 \%$ of the participants reported having an "excellent" experience 
Table 6 Percent agreement in obstruction risk between paper and each alternate mode of administration by mode pair

\begin{tabular}{lllllll}
\hline $\begin{array}{l}\text { P-based with } \\
\text { alternate } \\
\text { mode }\end{array}$ & $\begin{array}{l}\text { Obstruction } \\
\text { risk, both } \\
\text { modes n (\%) }\end{array}$ & $\begin{array}{l}\text { No obstruction } \\
\text { risk, both } \\
\text { modes n (\%) }\end{array}$ & $\begin{array}{l}\text { Agreement } \\
\text { n (\%) }\end{array}$ & $\begin{array}{l}\text { Obstruction } \\
\text { risk, paper } \\
\text { mode only n (\%) }\end{array}$ & $\begin{array}{l}\text { Obstruction } \\
\text { risk, alternate } \\
\text { mode only n (\%) }\end{array}$ \\
\hline W & $34(70.8 \%)$ & $12(25.0 \%)$ & $46(95.8 \%)$ & $0(0.0 \%)$ & $2(4.2)$ & 0.89 \\
IVRS & $28(62.2 \%)$ & $12(26.7 \%)$ & $40(88.9 \%)$ & $5(11.1 \%)$ & $0(0.0 \%)$ & $3(7.1 \%)$ \\
I & $31(73.8 \%)$ & $5(11.9 \%)$ & $36(85.7 \%)$ & $3(7.1 \%)$ & 0.75 \\
\hline
\end{tabular}

Abbreviations: I, interviewer; IVRS, interactive voice response system; P, paper; W, Web.

using the P- (60.3\%), W- (68.0\%), IVRS- (56\%), and I-based (73.5\%) modes of administration. Only 1 participant reported having a "poor" overall experience on the IVRS-based mode and 6 participants reported having a "fair" overall experience (ie, 3 on the P-based mode, 1 on the W-based mode, and 1 on the IVRS-based mode).

The multiple administration modes were generally well understood and deemed acceptable for use. Most participants indicated that they encountered no difficulties completing the LFQ on any of the platforms provided.

Of the 149 participants, only 18 indicated that they had any problems when completing the LFQ. Of those, the majority $(n=13)$ were in relation to the IVRS-based mode. The most commonly reported complaint was that the IVRS system required respondents to wait until all answer choices were given for each question before the system would allow the selection of a response; however, no one indicated that they would be unwilling to complete the LFQ via an IVRS-based system, especially given the brevity of the measure.

The last item in the satisfaction survey asked respondents which mode they preferred out of the two LFQ presentations. Over $80 \%$ of the participants who were given the W-based mode preferred it over the P-based mode. Approximately $60 \%$ of participants assessed by an interviewer preferred the I-based mode over the P-based mode. However, of those given the IVRS-based mode, only $25.5 \%$ participants selected it over the P-based mode; $61.7 \%$ reported preferring the P-based more than the IVRS-based mode.

\section{Discussion and conclusions}

The LFQ used in this study is a case-finding tool that had been developed and validated previously to identify symptomatic patients who need further evaluation. ${ }^{2,5}$ Spirometry (the gold standard for diagnosis) has not been shown to be as practical a tool because patients are unlikely to present with respiratory symptoms at the initial phases of COPD. Spirometry is physically burdensome on patients and rarely used in primary care. Early symptoms of COPD are often missed; therefore, COPD remains largely underdiagnosed. ${ }^{3,4}$
The LFQ is composed of a series of simple questions that may aid a physician in a clinical encounter, in a standardized manner. Our study examined properties between different modes of the LFQ. Before this study, the LFQ was validated only in the paper mode, limiting the administration and availability of the measure. Our objective was to validate an electronic (ie, Web-administered) mode, an interviewer-administered, and a telephone (IVRS) mode to allow for broader use and dissemination of LFQ in both study design and scope. In instrument development, whenever a version other than the validated version of a tool is used, additional examination of the psychometric properties of that alternate version may be warranted to ensure that the properties of the new form are similar to the properties of the original version. Therefore, we conducted a comprehensive psychometric examination of instrument properties for alternate modes of LFQ. ${ }^{10,11}$

This study showed a great degree of concordance in alternate forms of the LFQ with the paper version. The absence of floor and ceiling effects ensured that transitioning from P-based administration mode to the other modes retained the measurement properties of the original P-based version. Average LFQ item-level means were fairly consistent across modes, and there was no evidence to support sequence effects (whether taking one mode before the other influenced participant responses in the subsequent mode). LFQ scores over 7 days were stable between assessments, and the P-based and alternate administration modes showed exceptionally good agreement as evidenced by high ICCs. In addition, the classification of risk of AO using the LFQ cut point remained unchanged, for the most part, between paper and alternate modes. The satisfaction results indicated that participants were highly satisfied across all modes, indicating that patients are likely to respond favorably to requests to answer questions on the LFQ irrelevant of the mode of administration.

Together, the patient satisfaction insights and psychometric evidence indicate a high level of acceptance and comparability for the LFQ regardless of administration mode. Therefore, offering multiple choices or modes of data collection may improve respondent willingness to participate, 
increase response rates, and provide for earlier intervention for patients at risk for AO. Additionally, wider accessibility of the, LFQ may encourage and facilitate dialog between patients and health care providers regarding the risk of COPD. Although the results of this study cannot be extrapolated to other questionnaires or to uses of the LFQ for purposes other than as a case-finding tool, it does provide important insights into the lack of effect of mode in this population.

The LFQ is an important case-finding tool to aid primary care physicians in further evaluating symptomatic patients at risk of AO. Alternate modes of administration for this tool will further facilitate its implementation. This study has shown that there is no loss in psychometric properties of LFQ, irrespective of the mode of administration (ie, P-, W-, IVRS-, or I-based). As of the date of this publication, we are not aware of any tools that have been validated in alternate versions for use in COPD. Additionally, these alternate modes can be combined with multiple language versions. To date, the LFQ has also been cross-culturally adapted and translated into Mandarin Chinese for China, Japanese for Japan, Korean for Korea, Russian for Russia, Spanish for the United States, and Vietnamese for Vietnam. With the projection that COPD will rank fifth in burden worldwide by 2020 , tools to increase awareness and earlier diagnosis are needed to improve patient outcomes. Physicians in primary care settings are not likely to perform spirometric evaluations on all of their patients, or even on the subset of patients (smokers) due to the expense and inconvenience of including those tests in usual care. If the LFQ is provided in primary care settings and made available online, patients may be more likely to discuss their risk for COPD after completing the questions on the LFQ. Based on his or her score, a patient may request spirometry or be more likely to perform spirometry, leading to earlier diagnosis and treatment interventions for patients at risk for developing COPD.

\section{Acknowledgment}

The authors thank Alyssa Dallas, Margaret Mathes, and Candace Webster of RTI Health Solutions for their editorial and graphical assistance. GlaxoSmithKline funded this study (ADC001HO)

\section{Disclosure}

Anand Dalal is an employee of GlaxoSmithKline and owns company stock. Carla DeMuro-Mercon, Sandy Lewis, Lauren Nelson, Theresa Gilligan, and Lori McLeod are employees of RTI Health Solutions. GlaxoSmithKline funded this study. RTI Health Solutions, sponsored by GlaxoSmithKline, conducted research in support of this study.

\section{References}

1. Global Initiative for Chronic Obstructive Lung Disease (GOLD). Guidelines: global strategy for diagnosis, management, and prevention of COPD. Available from: http://www.goldcopd.com/Guidelineitem.asp? $11=2 \& 12=1 \&$ intId=2003. Updated 2009 Dec. Accessed 2010 Sep 9.

2. Hanania NA, Mannino DM, Yawn BP, Mapel DW, Martinez FJ, Donohue JF. Predicting risk of airflow obstruction in primary care: validation of the lung function questionnaire (LFQ). Respir Med. 2010;104(8):1160-1170.

3. Clinical consensus in COPD. Experts stress COPD under-diagnosis. Medwire News. 2007 Mar 7. Available from: http://www.medwire-news. md/48/65026/Respiratory/Experts_stress_COPD_under-diagnosis. html. Accessed 2010 Sep 9.

4. Clotet PJ, Gómez-Arbonés X, Ciria C, Albalad JM. Spirometry is a good method for detecting and monitoring chronic obstructive pulmonary disease in high-risk smokers in primary health care. Arch Bronconeumol. 2004;40:155-159.

5. Crater G, Dalal AA, Gilsenan A, et al. Prevalence of chronic obstruction pulmonary disease in subjects with a history of cigarette smoking assessed in a primary care setting. Paper presented at: 2010 American Thoracic Society International Conference; 2010 May 14-19; New Orleans, LA. Available from: https://cms.psav.com/cAbstract/itinerary/ title.html. Publication number: A5953. Accessed 2010 Sep 9.

6. Streiner DL, Norman GR. Health Measurement Scales: A practical guide to their development and use. 2nd ed. New York, NY: Oxford University Press; 1995.

7. Landis RJ, Koch GG. The measurement of observer agreement for categorical data. Biometrics. 1977;33:159-174.

8. Nunnally JC, Bernstein IH. Psychometric Theory. 3rd ed. New York, NY: McGraw-Hill; 1994.

9. World Health Organization, Expert Committee on Physical Status. Physical Status: The use and interpretation of anthropometry. Technical Report Series Nr 854. Geneva, Switzerland: World Health Organization; 1995.

10. US Department of Health and Human Services. Food and Drug Administration. 2009 Dec. Guidance for industry patient-reported outcome measures: use in medical product development to support labeling claims. Available from: http://www.fda.gov/downloads/Drugs/ GuidanceCom plianceRegulatoryInformation/Guidances/UCM193282. pdf. Accessed 2010 Sep 9.

11. Coons SJ, Gwaltney CJ, Hays RD, et al. Recommendations on evidence needed to support measurement equivalence between electronic and paper-based patient-reported outcome (PRO) measures: ISPOR ePRO Good Research Practices Task Force Report. Value Health. 2009:12(4):419-429.
International Journal of COPD

\section{Publish your work in this journal}

The International Journal of COPD is an international, peer-reviewed journal of therapeutics and pharmacology focusing on concise rapid reporting of clinical studies and reviews in COPD. Special focus is given to the pathophysiological processes underlying the disease, intervention programs, patient focused education, and self management protocols.

\section{Dovepress}

This journal is indexed on PubMed Central, MedLine and CAS. The manuscript management system is completely online and includes a very quick and fair peer-review system, which is all easy to use. Visit $\mathrm{http}: / / \mathrm{www}$.dovepress.com/testimonials.php to read real quotes from published authors. 